\title{
LES ARMES D'APPARAT ET LES PIÈCES D'EQUIPEMENT EQUESTRE DU PRESTIGE DANS LA RÉGION DU DNIEPR MOYEN ET SUPÉRIEUR A L'ÉPOQUE POST-HUNNIQUE
}

\author{
M I C H E L K A Z A N S K I
}

\begin{abstract}
Representative Weapons and Luxury Components of Equestrian Equipment in the Middle and Upper Dnieper Area in the post-Hunnic Period. Archaeological findings of prestigious nature of the post-Hunnic era (second half of the $5^{\text {th }}$-middle of the $6^{\text {th }}$ c.) and items that testify to the spread of the 'military' material culture in the Upper and Middle Dnieper basin, on the territory of the Slavic cultures of Kolochin and Penkovka are considered. These findings enable to determine the geography of possible centres of power. One of them was on the left bank of the Dnieper, somewhere between the Upper Psel and the Desna, and the second on the right bank of the Dnieper, in the Ros-Tiasmina area. Findings of prestigious weapons (helmets of the Baldenheim type, an early Byzantine sword and a 'prestigious' horse bridle) and characteristic elements of a belt set indicate the formation of a military elite. Data from written byzantine sources of 530-570 confirm the existence of military leaders and professional soldiers in the Slavic society.
\end{abstract}

Keywords: Dnieper, Slavs, post-Hunnic period, warlike elites.

Notre but est de présenter ici les découvertes archéologique "prestigieuses » à caractère militaire - armes et pièces de harnachement - dans la région du Dniepr moyen et supérieur à l'époque post-hunnique (deuxième moitié du $\mathrm{V}^{\mathrm{e}}$-milieu du $\mathrm{VI}^{\mathrm{e}} \mathrm{s}$.). A cette époque, les sites de la civilisation de Kolotchin (dernière synthèse: Oblomsky 2016) occupent la partie septentrionale de cette région, essentiellement dans la zone forestière, ceux de la civilisation de Penkovka (monographie de synthèse toujours en vigueur: Prihodniuk 1998) se situent plus au Sud, dans une bande de steppe forestière (fig. 1). Ces deux civilisations, très similaires, font partie des Slaves. Si ce n'est pas déterminé pour la civilisation de Kolotchin, faute de témoignages de sources écrites, la population de la civilisation de Penkovka est associée aux Antes, proches parents des Sclavènes (les Slaves à proprement parler des auteurs anciens, porteurs de la civilisation de Prague, plus à l'Ouest). Ces Antes sont décrits ou mentionnés par Jordanès, Procope de Césarée, Ménandre, Michel le Syrien, Maurice et d'autres auteurs du $\mathrm{VI}^{\mathrm{e}}-\mathrm{VII}^{\mathrm{e}} \mathrm{s}$. (à propos de la localisation géщgraphique des Antes voir: Kazanski 2013).

Les découvertes d'armes et de harnachements prestigieux de l'époque post-hunnique sont attestées dans la région du Dniepr dans deux zones:

- Dniepr - rive gauche, entre les rivières Psel et Desna (civilisation de Kolotchin; fig. 1: 1-4);

- Dniepr - rive droite, entre les rivières Stugna et Tiasmina (civilisation de Penkovka; fig. 1: 5, 6).
Examinons d'abord les découvertes dans la zone de la civilisation de Kolotchin:

Kartamyschevo (Картамышево), district d'Oboian, province de Koursk, Russie (fig. 1: 1). Une épée byzantine $d u \mathrm{~V}^{\mathrm{e}}$-première moitié $d \mathrm{u} \mathrm{VI}^{\mathrm{e}} \mathrm{s}$. y a été fortuitement mise au jour (Kazanski 2015, 49; Radiush 2012, 141, 142). Elle est du type dit pontique, avec une large garde en bronze (fig. 2; Menghin 1995, 176-186). Dans le Barbaricum, entre le Caucase et le Rhin, ces épées sont typiques des tombes de « chefs militaires » (Kazanski 2001).

Région de Klimovo (Кдимово), district de Klimovo, province de Briansk, Russie (fig. 1: 2). Il s'agit d'une découverte fortuite de fragments en bronze doré et de morceaux de cotte de maille, provenant d'un casque du type Baldenheim (fig. 3), ainsi que de mors ornés des têtes d'oiseaux (fig. 4: 2, 3; Kazanski 2018, 86-89; Radiush 2014, 43, 44, fig. 5; Schinakov 2015; Schinakov/Gratchev 2014). Les casques du type Baldenheim sont bien connus en Europe, aussi bien en Occident mérovingien que dans les pays du Danube et dans la zone méditerranéenne, depuis la deuxième moitié-fin du Ve $\mathrm{s}$. à la fin du VI $\mathrm{s}$. Cependant le nombre le plus important de découvertes correspond à la fin du $\mathrm{V}^{\mathrm{e}}$-première moitié $\mathrm{du}$ $\mathrm{VI}^{\mathrm{e}} \mathrm{s}$. Ces casques sont au moins en partie d'origine byzantine (Bavant 1990 252-254; 2008; Kazanskil Mastykova/Périn 2002, 170; Quast 1993, 39-42; Werner 1988). Ainsi, les fragments d'un casque découverts à Héraclée, dans les Balkans, portent des marques 


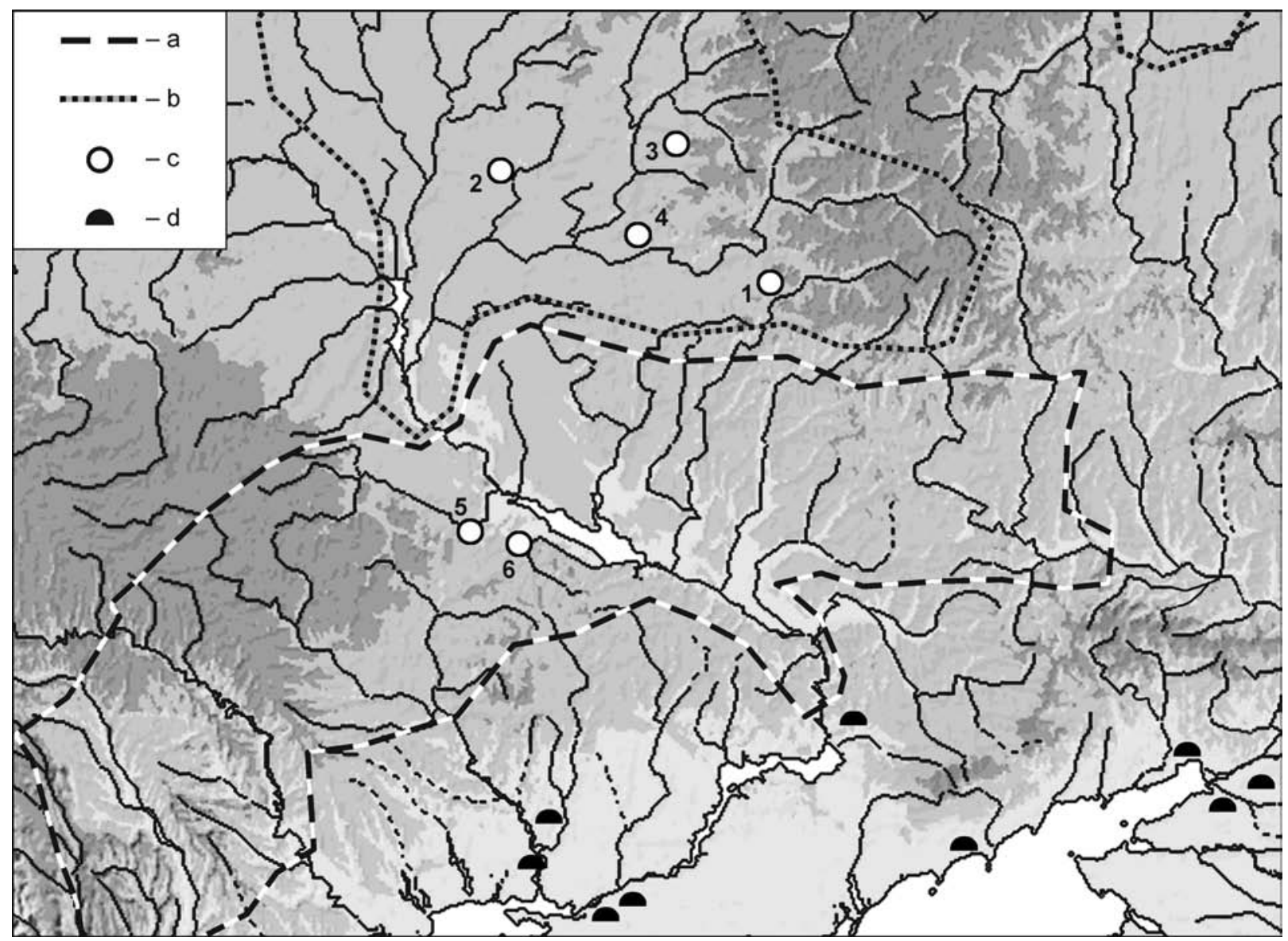

Fig. 1. Découverts «prestigieuses » à caractère militaire de l'époque post-hunnique dans a région du Dniepr supérieur et moyen. 1 - Kartamyschevo; 2 - Klimovo; 3 - Boldyjsky Les; 4 - Gloukhov; 5 - Babitchi; 6 - Tcherkassy. Légende: a - la frontière de la civilisation de Kolotchin; $\mathrm{b}$ - la frontière de la civilisation de Penkovka; $\mathrm{c}$ - découverts prestigieuses à caractère militaire; $\mathrm{d}$ - sites des nomades de l'épouqe post-hunnique.

de fabrications avec des images d'Anastase et de Justin Ier (Maneva 1987). En Europe, ces casques sont peu nombreux et proviennent surtout des sépultures de chefs (Quast 1993, 131-133; Vogt 2006). En Europe orientale, jusqu'à une époque récente, ils étaient quasi-absents. On ne pouvait citer que la découverte d'une forme similaire, du type Bretzenheim, provenant de Kertch, en Crimée (Glad 2009 fig. 7: 17; Post 1953, fig. 23; Quast 1993, Liste 2, n 55, 56). D'autre part, un casque du type Baldenheim a été mis au jour en Russie centrale, dans la nécropole de Tsaritsyno (Царицыно; le district de Kasimov, de la province de Riazan), appartenant aux Finnois de la civilisation dite de Riazan-Oka (Akhmedov/Birkina 2017, fig. 4). Or, comme nous allons le voir, le casque de Klimovo n'est pas unique dans la région du Dniepr.

En ce qui concerne les pièces de harnachement provenant de la même découverte (fig. 4), notamment les mors à tiges ornées de têtes aviformes, ils sont typiques, eux aussi, des tombes de chefs de la deuxième moitié du $\mathrm{V}^{\mathrm{e}}$-première moitié du VIe $\mathrm{s}$. dans une large zone, allant du Caucase jusqu'à la Suède septentrionale et la France. Citons à titre d'exemple les découvertes à Bylym-Kudinetovo, tumulus 14 en Kabarda-Balkarie, à Högom, tumulus 2, en Norrland ou encore à Charleville-Mézières, tombe 68, dans les Ardennes (Akhmedov 2002; Kazanski 2016).

Boldyzhsky Les, rivière Navlia (Болдыжский Лec, Нав $九$ ), district de Navlia, province de Briansk, Russie (fig. 1: 3). Une autre découverte fortuite de fragments de casque du type Baldenheim (paragnathides en bronze doré, portant un décor en écailles) est attestée dans la même région que celle de Klimovo (fig. 5; Radiush 2014, 43, 43, fig. 4). Ce type de décor est bien représenté sur les casques du type Baldenheim (Quast 1993, fig. 18; Werner 1988, fig. 1-3). La deuxième découverte d'un casque du type Baldenheim dans la même région est une preuve que leur apparition ici n'est pas un hasard. 


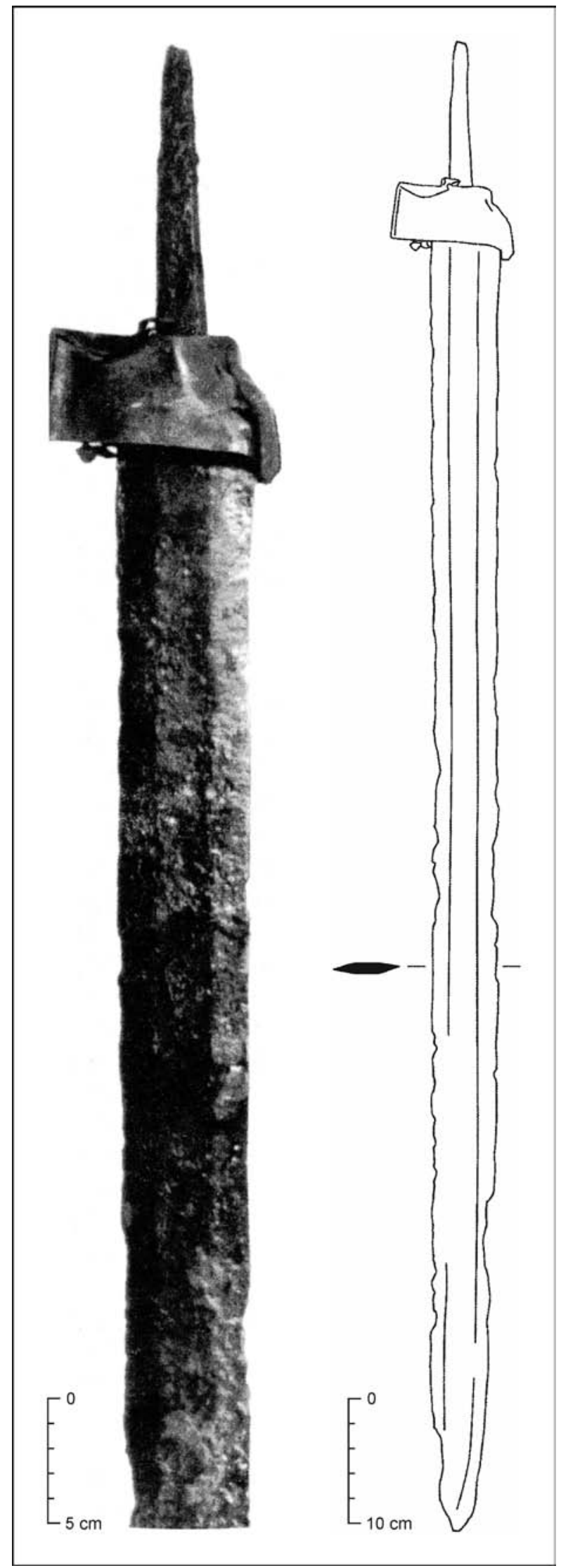

Fig. 2. Kartamyishevo. L'épée (d'après Radiush 2012, fig. 2: 2).
Région de Glukhov (Гиухів), district de Glukhov, province de Sumy, Ukraine (fig. 1: 4). Des fouilleurs clandestins ont découvert un trésor (?) contenant des pièces de harnachement, notamment des mors et des garnitures de courroies (fig. 6; Radiush 2014, 45). Les mors à tige godronnée et à l'extrémité en forme de plaque allongée (fig. 6: 1, 2), ainsi que les plaques-boucles à plaque triangulaire (fig. 6: 11, 12) sont typiques du harnachement à l'époque post-hunnique en Europe de l'Est (Kazanski/Mastykova 2010, 95, 98). Les appliques ovales, ornées de cabochons (fig. 6: 26, 27), sont proches de celles provenant d'une tombe d'un chef hunno-bulgare de la deuxième moitié du $\mathrm{V}^{\mathrm{e}}$-début du $\mathrm{VI}^{\mathrm{e}} \mathrm{s}$. à Morskoï Tchoulek (Морской Чулек), près de l'embouchure du Don (Kazanski 2010, fig. 9: 2; 2017a, fig. 12: 12) et de celles découvertes dans une tombe hunno-bulgare en Crimée, dans la nécropole Neïzatz (Нейзац), sépulture 114 (Hrapunov/Kazanski 2016, fig. 15: 4). Les appliques circulaires à décor cloisonné (fig. 6: 22, 23), rappellent celles provenant de la tombe « princière » d'Apahida II en Transylvanie (Harhoiu 1998, tab. LXIV: 1-10; L'Or des princes barbares 2000, kat. $\left.n^{\circ} 29: 22,23\right)$. Les appliques à quatre têtes d'oiseaux (fig. 6: 37-40) sont comparables aux appliques de harnachement provenant de Verhniaia Rutha, dans le Caucase du Nord (Abramova 1997, fig. 57: 3).

Une des plaques-boucles provenant de cette découverte (fig. 6: 25) est un prototype direct des pseudo-boucles de la deuxième moitié du $\mathrm{VI}^{\mathrm{e}}-\mathrm{VII}{ }^{\mathrm{e}}$ s. (voir à leur propos: Gavrituhin 2001). Enfin, une plaque-boucle en B (fig. 6: 19) est très proche de celles du Caucase du Nord et de la Russie centrale du VI e s. ancien (Voir: Mastykoval Kazanski/Saprykina 2016, 14). Tout ceci nous permet d'attribuer la découverte de Gluhov à la première moitié-milieu du VI ${ }^{\mathrm{e}} \mathrm{s}$.

Babitchi (Бабичи), ancien district de Tcherkassy (avant 1917), Ukraine (fig. 1: 5). On a mis au jour au XIXe s., dans deux tumuli, deux appliques de selle en tôle d'or, portant un décor estampé en écaille, deux boucles, une rectangulaire et une en $B$, un ferret avec une extrémité en forme de croissant et une petite tige, tous issus d'un harnachement en or massif (fig. 7: B; Kazanski 2017a, fig. 2: 13-17; 2018, $92,93)$. On ne sait pas si ces objets proviennent de la même sépulture. Selon I. Zaseckaja, les appliques de selle (fig. 7: B: 8) sont d'une forme caractéristique de la deuxième moitié du $\mathrm{V}^{\mathrm{e}}$-première moitié du VIe s., du type IVb (Zaseckaja 1999, 348-350, fig. 7). Il est possible que cette découverte appartienne aux nomades hunno-bulgares de la steppe pontique (Kazanski 2017a, 66). Cependant cette sépulture est très éloignée de la zone de diffusion des tombes hunno-bulgares de l'époque post-hunnique et 


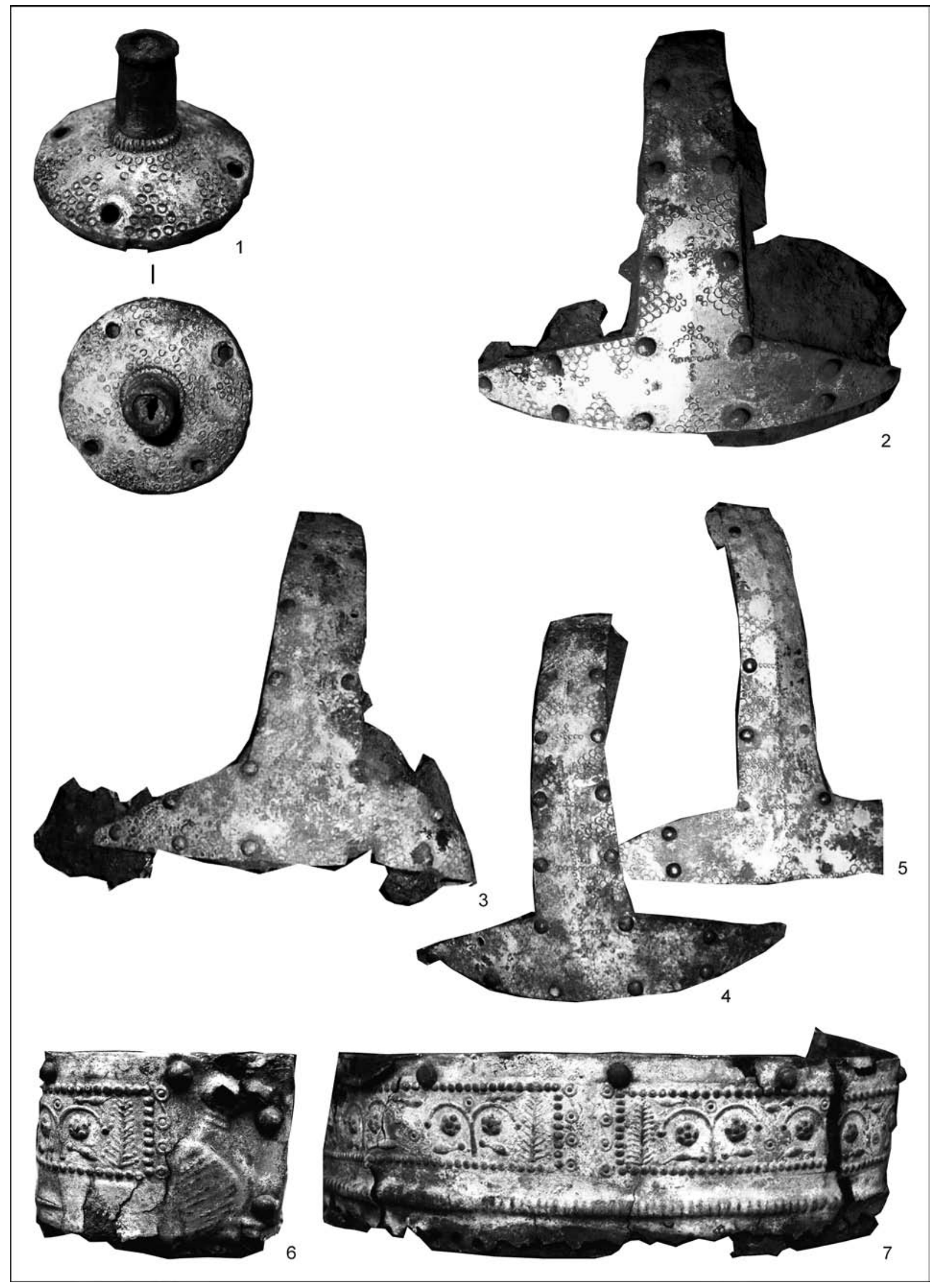

Fig. 3. District de Klimovo. Fragments de casque (d'après Radiush 2014, fig. 5: 1-6). 


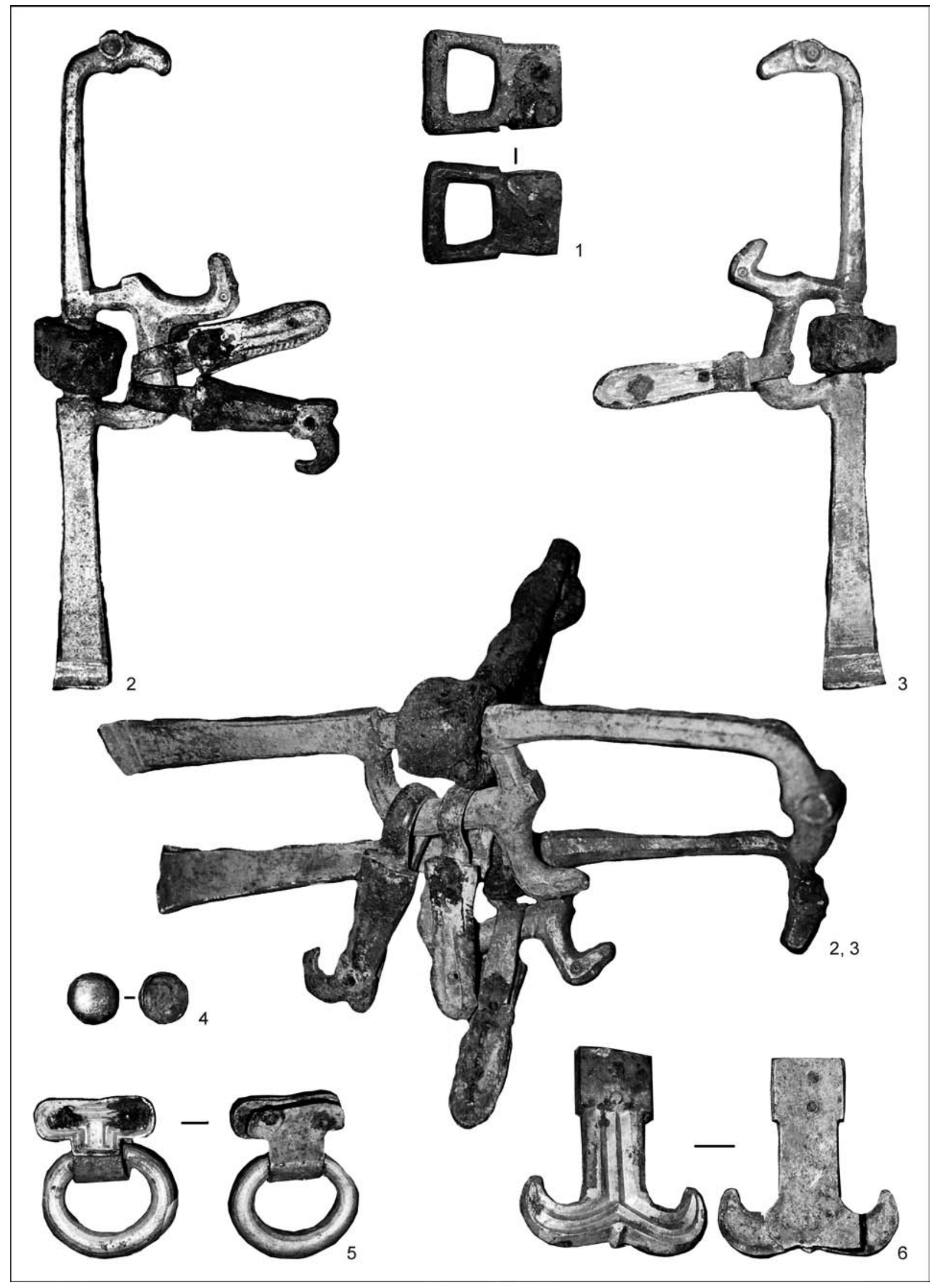

Fig. 4. District de Klimovo. Pièces de harnachement (d'après Radiush 2014, fig. 5: 1, 7; Shinakov/Gratchev 2014, fig. 1; 5; 16). 


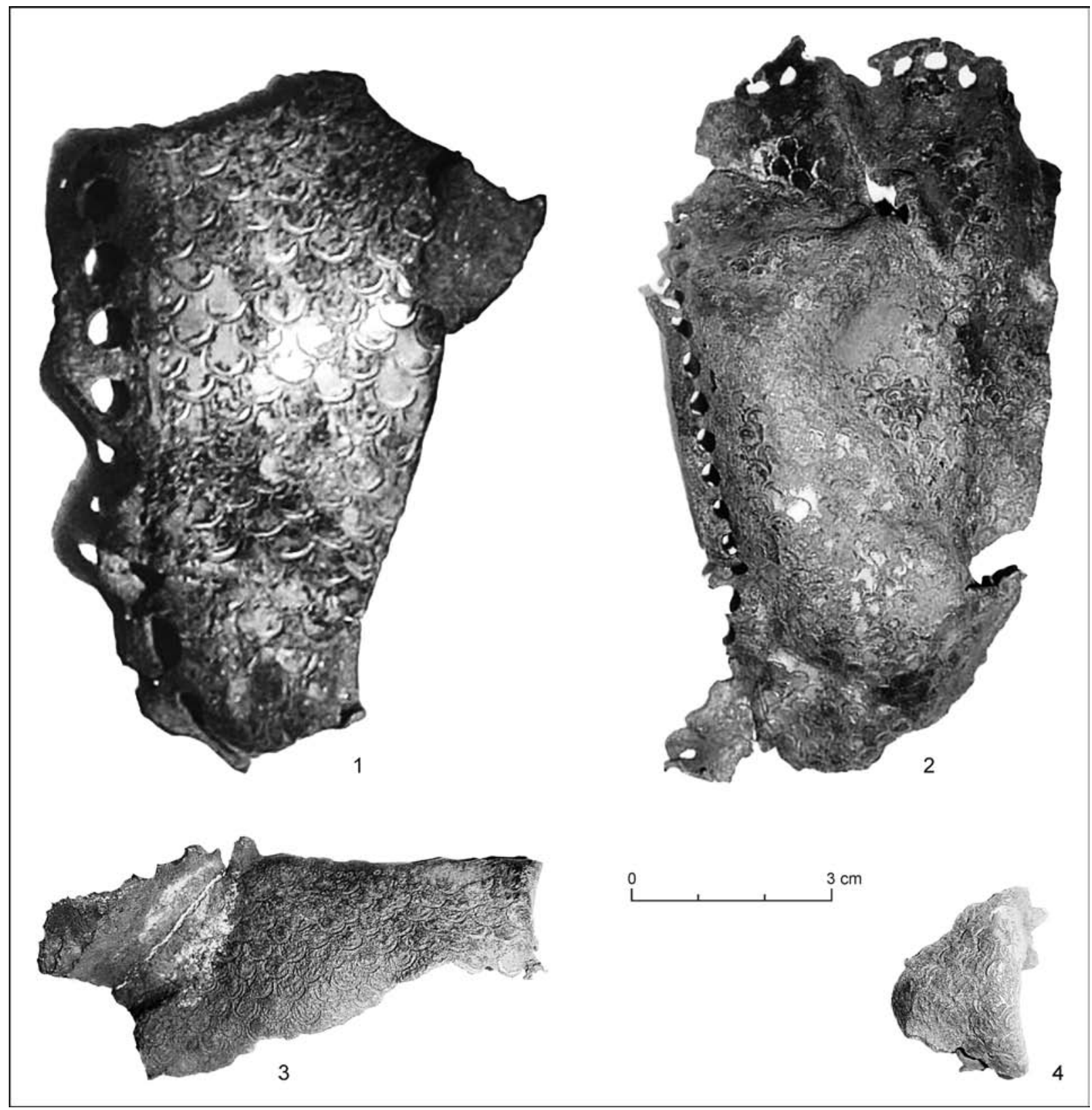

Fig. 5. Boldyjsky Les. Fragments de casque (d'après Radiush 2014, fig. 4).

se situe en plein territoire de la civilisation de Penkovka (fig. 1). D'autre part, la coutume du dépôt de selles dans des tombes de chefs barbares sédentaires est bien attestée au $\mathrm{V}^{\mathrm{e}} \mathrm{s}$. dans l'espace ponto-danubien; citons à titre d'exemple les fameuses tombes d'Apahida, de Conceşti et de Diurso (Kazanski 2017b, 204, 205).

Province de Tcherkassy (Черкаси), Ukraine (fig. 1: 6). Fragments d'un casque du type Baldenheim (fig. 7: A); le lieu exact et les circonstances de la découverte sont inconnus. On sait que le casque a été mis au jour à une profondeur d'environ $40 \mathrm{~cm}$, dans la masse d'une cotte de maille corrodée, qui pesait autour de $20 \mathrm{~kg}$ (Kazanski 2018, 93, 94; Radiush 2019, 42, 43).

D’autre part, dans la région du Dniepr, à l'époque post-hunnique, on connait bien des pièces métalliques, essentiellement des garnitures de ceinture, provenant du costume masculin/militaire et de l'équipement guerrier (fig. 8). Leur attribution est faite d'après la présence de ces objets dans des tombes «masculines/militaires» dans d'autres régions, par exemple dans des nécropoles de la civilisation finnoise de Riazan-Oka, dans des tombes 


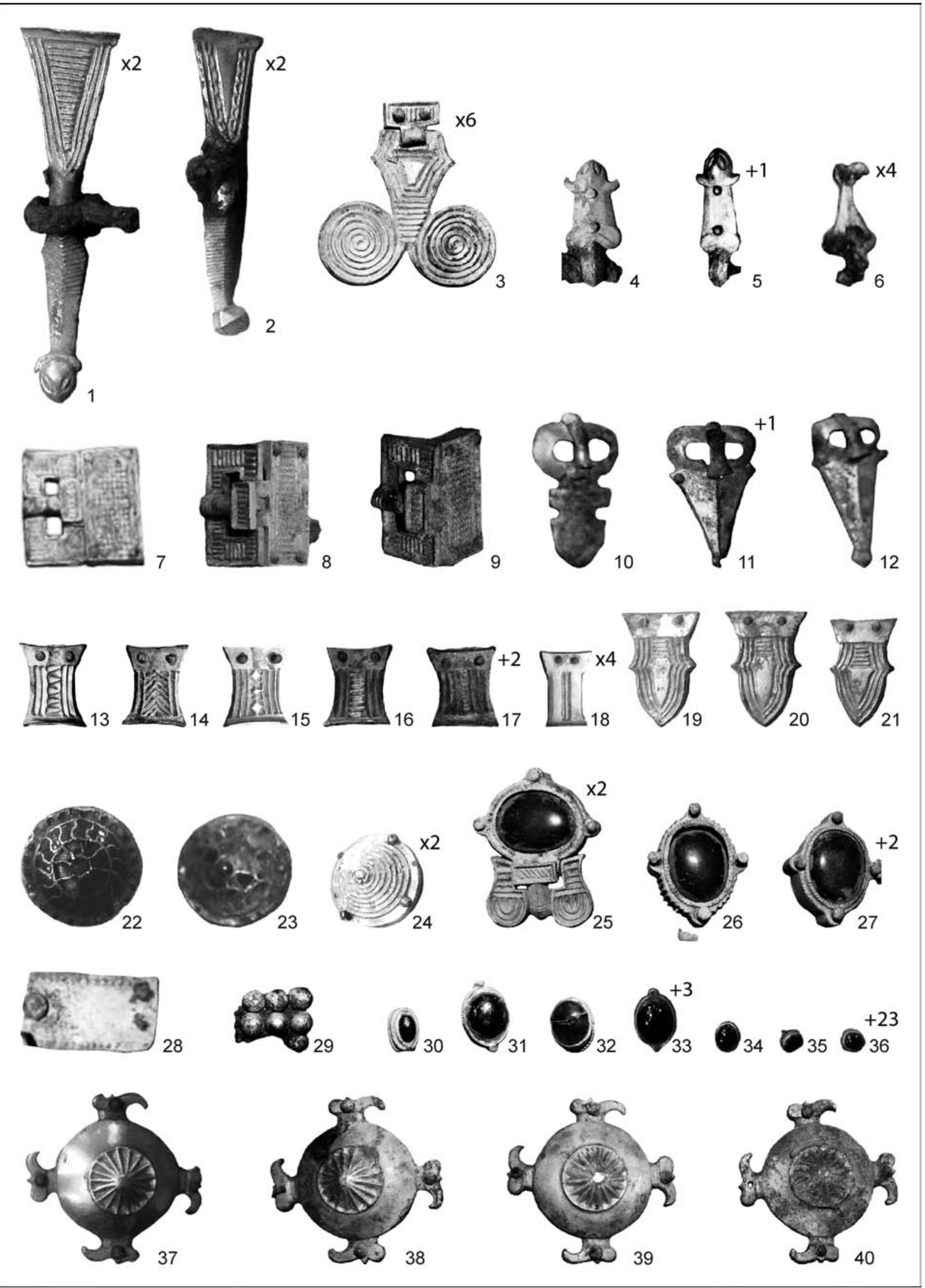

Fig. 6. District de Gloukhov. Trésor? (d'après Kazanski 2018, fig. 7). 


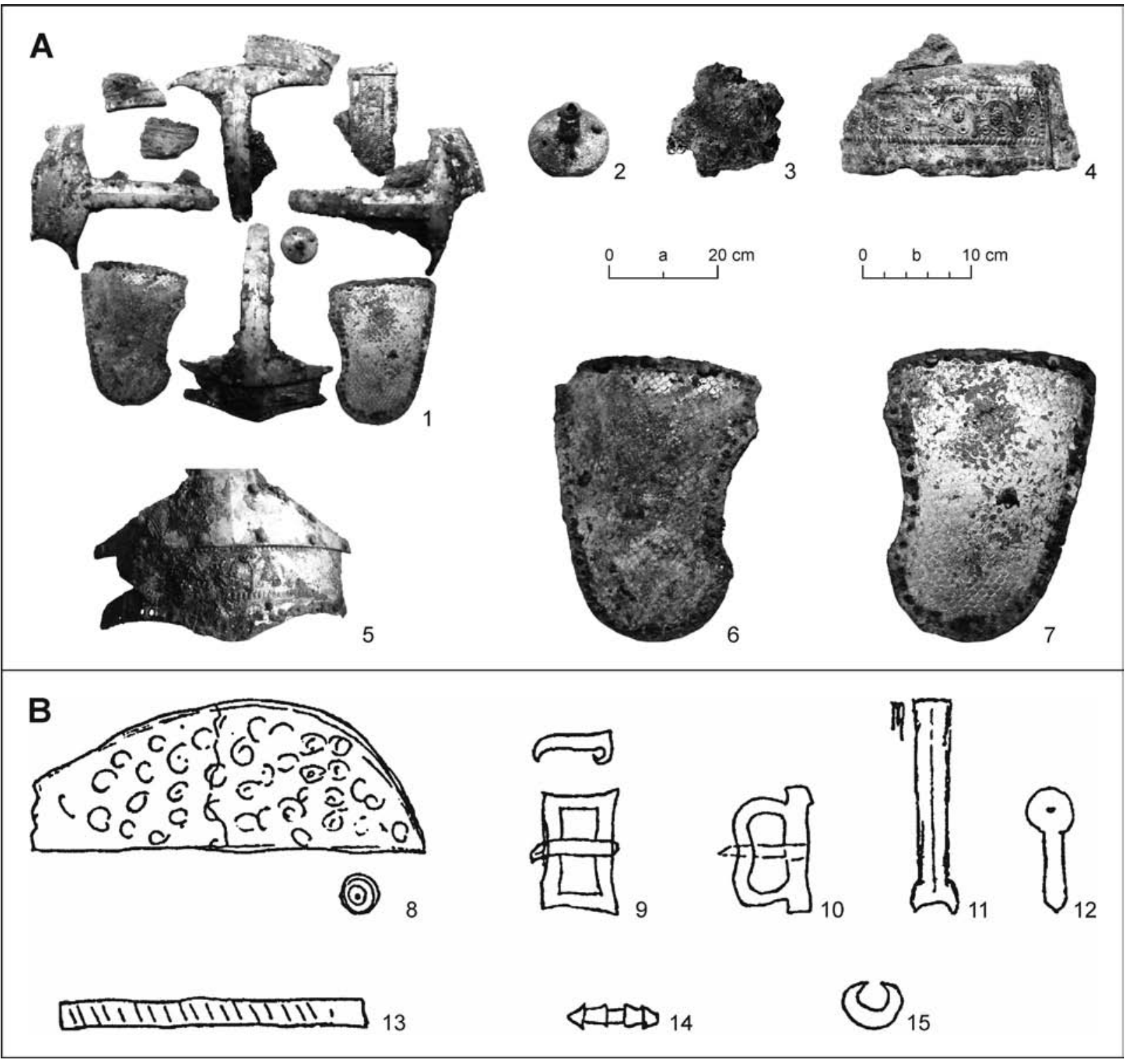

Fig. 7. Découvertes dans la région du Dniper-rive droite. A - Babitchi. Objets venant d'un tumulus (d'après Kazanski 2017a, fig. 2: 13-17); B - Province de Tcherkassy. Fragments de casque (d’après Kazanski 2018, fig. 10). Échelle: a -1; b - 2-7; sans échelle $-8-15$.

des Baltes orientaux ou de Germains danubiens. Il s'agit notamment d'appliques en X (fig. 8: A: 4, 5), largement répandues en Europe orientale et centrale (Akhmedov 2014, 141) et de boucles ovales godronnées (fig. 8: A: 1-3), qui, en Europe de l'Est, sont attestées uniquement dans des tombes masculines (Kazanski 1999, 207).

Dans la partie nord de la région en question (fig. 8: B), on connaît des plaques-boucles et des pendentifs à anneau ovale et plaque réniforme (fig. 8: A: 6-8) de la deuxième moitié du $\mathrm{V}^{\mathrm{e}}$-première moitié du VIe s. (voir par ex. Legoux/Périn/Vallet 2016, $n^{\circ}$ 141, 142; Werner 1953, 323-329), qui, en Europe centrale et occidentale, sont typiques avant tout du costume masculin prestigieux, même si on les trouve parfois dans des tombes incontestablement féminines. Les plaques-boucles du Haut-Dniepr ont une particularité : leur plaque est ornée d'une ou deux têtes aviformes (Ambroz 1970, fig. 1: 1, 2; Kazanski 2018, 100). Les éléments de ceinture, ornés de deux têtes aviformes ou zoomorphes, disposées face à face, sont également caractéristiques de la région du Haut Dniepr et de la rive gauche du Dniepr (Ambroz 1970, fig. 1: 6; Akhmedov 2014, 143-151). Dans cette région, ces pièces proviennent soit de niveaux d'habitats, soit de découvertes fortuites, mais leur appartenance à la garniture des ceinturons masculins est confirmée par des 


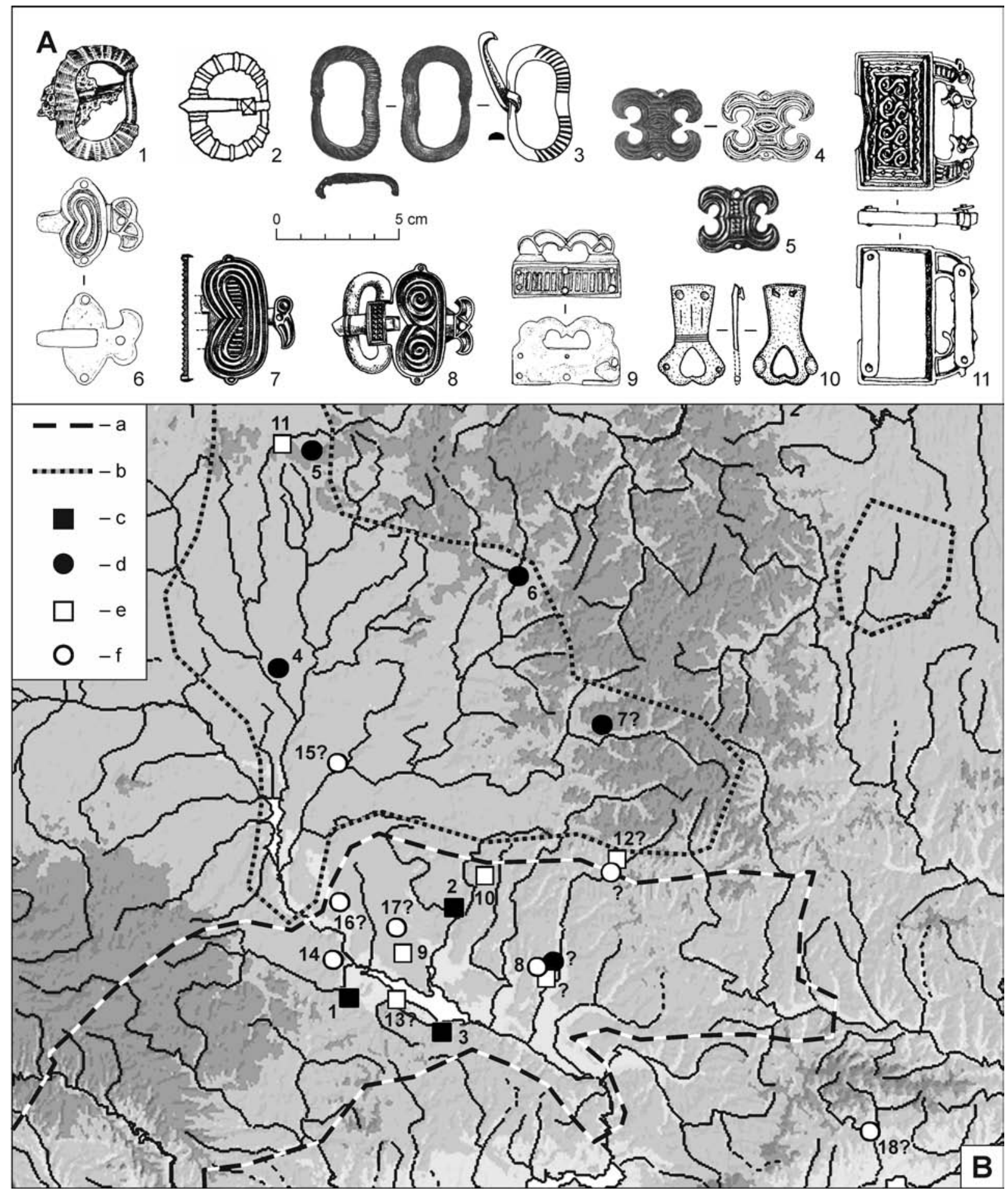

Fig. 8. Eléments de garnitures de ceinturons de l'époque post-hunnique. A - Boucles, appliques et ferrets: 1 - Bolshaia Andrusovka; 2 -Sahnovka; 3 - Hakrivtsy; 4-Hmelna; 5 -Hongie; 6-province de Briansk; 7 - Hotyischa; 8 -Demidovka; 9 - province de Smolensk; 10 - Lipovskoe; 11 - Tchapaevka (d'après 1, 2 -Kazanski 1999, fig. 4: 1, 2; 3-Volodarec-Urbanovitch 2016, fig. 1: 2; 2: 3; 4 - Akhmedov 2014, fig. 8: 2; 5 - Titov/Èrdeli 1986, fig. 12: 11; 6 - Akhmedov 2014, fig. 8: 6; 7, 8 - Ambroz 1970, fig. 1: 1, 2; 9 - Akhmedov 2014, fig. 8: 10; 10 - Prihodniuk 1998, fig. 64: 3; 11 - Akhmedov 2014, fig. 8: 4. B - Diffusion des éléments de garnitures de ceinturons: 1 - Sahnovka; 2 - Hakrivtsyi; 3 - Bolshai Andrusovka; 4 - Hotyscha; 5 - Demidovka; 6 - province derinsk; 7 - province de Koursk; 8 - province de Poltava; 9 - Tchapaevka; 10 - Lipovskoe; 11 - province de Smolensk; 12 - province de Sumy; 13 - province de Tcherkassy; 14 - Hmelna; 15 - province de Tchernigov; 16 - Berezan; 17 - Zolotonoscha; 18 - province de Donetsk. Légende: $\mathrm{a}$ - la frontière de la civilisation de Kolotchin; $b$ - la frontière de la civilisation de Penkovka; c - boucles godronnées; $\mathrm{d}$ - boucles et pendentifs à plaque ovale, décorées de têtes aviformes; $\mathrm{e}$ - éléments de garnitures de ceinturons ornés de têtes aviforems et zoomorphes face à face; $\mathrm{f}$ - appliques en X. 
découvertes similaires dans des inhumations masculines, souvent avec des armes, dans des nécropoles finnoises de Russie centrale, plus à l'est (par ex. Akhmedov 2014, fig. 7: 2, 3, 6).

Ces découvertes "prestigieuses» dans le basin du Dniepr ont un caractère «militaire » prononcé : ce sont des casques de Baldenheim, une épée du type "pontique», des appliques de selle, etc. De toute évidence, ces objets appartiennent aux élites guerrières dirigeantes. Les découvertes d'armes d'appart, de harnachements de luxe et d'éléments de la «mode militaire» (garnitures de ceinture) permettent de parler d'existence d'une « mode militaire » à caractère international chez la population du Dniepr supérieur et moyen à l'époque posthunnique, de la deuxième moitié du $\mathrm{V}^{\mathrm{e}}$ au milieu $\mathrm{du} \mathrm{VI}^{\mathrm{e}} \mathrm{s}$. L'existence de zones particulières de diffusion de ces objets permet de localiser deux centres du pouvoir hypothétiques : l'un dans la région du Dniepr supérieur et de la rive gauche du Dniepr, quelque part entre les rivières Desna et Psel, sur le territoire de la civilisation de Kolotchin, et l'autre sur la rive droite du Dniepr, dans les bassins des rivières Ros et Tiasmina, c'est à dire dans la zone de la civilisation de Penkovka (fig. 1).
Quant aux sources écrites, elles donnent peu d'information sur l'existence d'élites guerrières chez les Sclavènes et les Antes. D'après les témoignages de Procope de Césarée sur les Antes dans les années 540 (Procope, Bel. Got. III.14.34) et l'information de Ménandre sur les relations avaro-antes (autour de 560) et avaro-sclavènes (578; Menandre, 6, 48), on peut conclure que les élites dirigeantes ayant des fonctions politiques et militaires existaient bien chez les peuples slaves. Les témoignages archéologiques que nous venons d'examiner pour les civilisations de Penkovka et de Kolotchin, confirment l'existence de telles élites.

Ces élites guerrières se manifestent probablement pour la première fois en 536-551, quand les Antes et les Sclavènes apparaissent au service de l'Empire d'Orient (par ex. Procope, Bel. Got. I.24.18-21) et quand une vague d'invasions slaves déferle sur les Balkans. On pense qu'ont participé à ces invasions, compte tenu de leur ampleur, un grand nombre de Slaves, venus parfois de loin (Teodor 1972; 1984). Il est bien possible que les armes romaines d'apparat, tels les casques de Baldenheim ou les épées «pontiques » tombent entre les mains des Slaves comme résultat de ces événements.

\section{BIBLIOGRAPHIE}

Abramova 1997 - M. Abramova : Rannie alany Severnogo Kavkaza III-V vv.n. è. Moskva 1997.

Akhmedov 2002 - I. Akhmedov: Cheek-pieces and elements of harness witch zoomorphic decoration in the Great Migrations period. In: J. Tejral (Hrsg.) : Probleme der frühen Merowingerzeit im Mitteldonauraum. Brno 2002, $11-30$.

Akhmedov 2014 - I. Akhmedov: Nekotorye indikatory kulturnyh vzaimodejstvij v drevnostiah riazanookskih finnov vtoroj poloviny $\mathrm{V}$-nachala VI v. In: A. Oblomskij (red.) : Problemy vzaimodejstvija naselenija Vostochnoj Evropy v èpohu Velikogo pereselenija narodov. Ranneslavïanskij mir 15. Moskva 2014, 138-177.

Akhmedov/Birkina 2017 - I. Akhmedov/N. Birkina: Chlemy iz mogilnika rïazano-okskih finnov u s. Caricyno (predvaritelnoe soobshenie). In: V. Rodinkova/ O. Rumïanceva (red.): Evropa ot Latena do Srednevekovija: varvarskij mir i rojdenie slavïanskih kultur. K 60-letiou A. M. Oblomskogo. Ranneslavïanskij mir 19. Moskva 2017, 235-248.

Ambroz 1970 - A. Ambroz : Ioujnye khudojestvennye sviazi naselenia verhnego Podneprovia v VI v. In: I. Kukharenko (red.): Drevnie slavïane $i$ ikh sosedi. Materialy $\mathrm{i}$ issledovania po arheologii SSSR 176. Moskva 1970, $70-74$.

Bavant 1990 - B. Bavant: Note annexe sur les fragments de casques $\mathrm{N}^{\circ} 257$ à 260. In: B. Bavant/V. Kondich/J.-M. Spieser (dir.) : Carichin Grad II. Le quartier Sud-Ouest de la ville haute. Belgrad - Rome 1990, 247-257.
Bavant 2008 - B. Bavant: Fragments des casques du type Baldenheim trouvés à Carichin Grad. Malanges de l'Ecole française de Rome. Moyen Âge 120/2, 2008, 327-353.

Gavrituhin 2001 - I. Gavrituhin: Èvolucija vostotchnoevropejskih psevdoprïajek. In: D. Stachenkov (red.): Kultury evrazijskih stepej vtoroj poloviny I tuisïatcheletija n.è. (iz istorii kostyouma). Tomus 2. Samara 2001, 31-86.

Glad 2009 - D. Glad: Origine et diffusion de l'équipement défensif corporel en Méditerranée orientale (IVe-VIII ${ }^{e}$ s.). BAR International Series S1921. Oxford 2009.

Harhoiu 1998 - R. Harhoiu: Die frühe Völkerwanderungszeit in Rumänien. Bucureşti 1998.

Hrapunov/Kazanski 2016 - I. Hrapunov/M. Kazanskij: Pogrebenija èpohi pereselenija narodov v mogilnike Nejzac. In: I. Hrapunov (red.) : Krym v sarmatskuyu èpohu (II v. do n. ̀̀.-IV v.n. è.) 2. Dvadcat let issledovanij mogilnika Nejzac. Simferopol 2016, 194-229.

Kazanski 1999 - M. Kazanski : L'armement slave du haut Moyen-Age ( $\mathrm{V}^{\mathrm{e}}-\mathrm{VII}$ e siècles). A propos des chefs militaires et des guerriers professionnels chez les anciens Slaves. Přehled výzkumù 39, 1999, 197-236.

Kazanski 2001 - M. Kazanski: Les épées «orientales» à garde cloisonnée du $\mathrm{V}^{\mathrm{e}}-\mathrm{VI}^{\mathrm{e}}$ siècle. In : E. Istvánovits/ V. Kulcsár (eds.) : International Connections of the Barbarians in the $1^{\text {st }}-5^{\text {th }}$ centuries $A$. D. Aszód - Nyíregyháza 2001, 389-418.

Kazanski 2010 - M. Kazanski: Les Hunnugours et le commerce de fourrure au VI' siècle. In: C. Theune/F. Biermann/R. Struwe/G. H. Jeute (Hrsg.) : Zwischen Fjorden 
und Steppe. Festschrift für Johan Callmer zum 65. Geburtstag. Internationale Archäologie. Studia honoraria 31. Rahden/Westf. 2010, 225-238.

Kazanski 2013 - M. Kazanski: The Land of the Antes according to Jordanes and Procopius. In: F. Curta/B.-P. Maleon (eds.): The steppe lands and the world beyond them. Studies in honor of Victor Spinei on his $70^{\text {th }}$ birthday. Iaşi 2013, $35-42$.

Kazanski 2015 - M. Kazanski : Voorujenie i konskoe snarïajenie slavïan V-VII vv. Stratum plus 5, 2015, 43-95.

Kazanski 2016 - M. Kazanski : Les mors de cheval à décor zoomorphe de l'époque des Grandes Migrations. Bulletin de liaison de l'Association française d'archéologie mérovingienne 40, 2016, 26-32.

Kazanski 2017a-M. Kazanski: Tombes des élites steppiques de l'époque post-hunnique dans la région pontique. Přehled výzkumu 58, 2017, 65-84.

Kazanski 2017b - M. Kazanski: On the Funerary Rite of a Hunnic-time "Princely" Burial of Concești. In : Na hranicích impéria. Extra fines Imperii. Jaroslavu Tejralovi k 80. narozeninám. Brno 2017, 197-208.

Kazanski 2018 - M. Kazanski : Prestijnye nahodki i centry vlasti postgunnskogo vremeni v Podneprove. Stratum plus 4, 2018, 83-118.

Kazanski/Mastykova 2010 - M. Kazanski/A. Mastykova: Khronologitcheskie indikatory drevnostej postgunnskogo vremeni na Severnom Kavkaze. Verhnedonskoj Arheologitcheskij sbornik 5, 2010, 93-104.

Kazanski/Mastykova/Périn 2002 -M. Kazanski/A. Mastykova/ P. Périn: Byzance et les royaumes barbares d'Occident au début de l'époque mérovingienne. In: J. Tejral (Hrsg.) : Probleme der frühen Merowingerzeit im Mitteldonauraum. Brno 2002, 159-194.

Legoux/Périn/Vallet 2016 - R. Legoux/P. Périn/F. Vallet: Chronologie normalisée du mobilier funéraire mérovingien entre Manche et Lorraine. Saint-Germain-en-Laye 2016.

Maneva 1987 - E. Maneva: Casque à fermoir d'Héraclée. Archaeologia Jugoslavica 24, 1987, 101-111.

Mastykova/Kazanski/Saprykina 2016 - A. Mastykova/ M. Kazanski/I. Saprykina: Pachkovskij mogilnik No. 1. Issledovanie materialov Pachkovskogo mogilnika No. 1. Tomus 2. Moskva - Sankt-Peterburg 2016.

Menandre - Menandre Protektor, Istorija. Perevod i kommentarij I. A. Levinskoj i S. R. Tohtasieva. In: L. Gindin/ G. Litavrin (red.): Svod drevnejchikh pismennykh izvestij o slavïanakh. Tomus I (I-VI vv). Moskva 1994, 311-356.

Menghin 1995 - W. Menghin: Schwerter des Goldgriffspathenhorizonts im Museum für Vor- und Frühgeschichte, Berlin. Acta Praehistorica et Archaeologica 26, 27, 1995, 140-191.

Oblomsky 2016 - A. Oblomsky: Kolochinskaija kultura. In: A. Oblomskij/I. Islanova (red.) : Rannesrednevekovye drevnosti lesnoj zony Vostochnoj Evropy (VI-VII vv.). Ranneslavïanskij mir 17. Moskva 2016, 10-134.
L'Or des princes barbares: Du Caucase à la Gaule Ve siècle après J.-C. Paris 2000.

Post 1953 - P. Post: Der kupferne Spangenhelm. Bericht der Römisch-Germanischen Kommission 34, 1953, 115-150.

Prihodniuk 1998 - O. Prihodniuk: Penkovskaija kultura. Voronej 1998.

Procope, Bel. Got. - J. Haury (ed.) : Procopii Caesariensis opera omnia. Vol. II. De bellis libri V-VIII. Leipzig 1963.

Quast 1993 -D. Quast: Die merowingerzeitlichen Grabfunde aus Gültlingen (Stadt Wildberg, Kreis Calw). Stuttgart 1993.

Radiush 2012 - O. Radiush: Voorujenie III-V vv. n. è. s tchernïahovskikh poselenij Kurskogo Posemija. Stratum plus 4, 2012, 139-155.

Radiush 2014 - O. Radiush: Chlemy perioda Velikogo pereselenija narodov iz Podneprovija. In : Voinskie tradicii $v$ arkheologitcheskom kontekste: ot pozdnego latena do pozdnego srednevekovia. Tula 2014, 40-53.

Radiush 2019 - O. Radiush: Prestijnoe voorujenie i dospeh V-natch. VI vv. na vostoke ranneslavïanskogo mira. In: I. Gavrituhin/C. Trifunović (red.) : Balkan, Podunavlje i Istochna Evropa u rimsko dobe i u ranom srednjem veku. Novi Sad 2019, 261-288.

Schinakov 2015 - E. Schinakov: Konskaija uprïaj i detali chpangenhelma "temnykh vekov" iz Brïanskogo Podesenija. In: E. Schinakov (red.) : Arheologicheskie issledovanija v evroregione "Dnepr" v 2013 g. Briansk 2015, 111-125.

Schinakov/Gratchev 2014 - E. Schinakov/S. Gratchev : Voorujenie i konskaia uprïaj gunno-germanskogo proishojdenija s territorii Brianskoj oblasti. In : Ejegodnik NII fundamentalnykh i prikladnykh issledovanij za $2013 \mathrm{~g}$. Briansk 2014, 100-112.

Teodor 1972 - D. G. Teodor: La pénétration des Slaves dans les régions du S-E de l'Europe d'après les données archéologiques des régions orientales de la Roumanie. Balcanoslavica 1, 1972, 29-42.

Teodor 1984 -D. G. Teodor : Origines et voies de pénétration des Slacves au sud du Bas-Danube (VI-VII ${ }^{\mathrm{e}}$ siècles). In: Villes et peuplement dans l'Illyricum protobyzantin. Rome 1984, 63-84.

Titov/Èrdeli 1986 - V. Titov/I. Èrdeli : Arheologiâ Vengrii, konec II tysâtcheletiâ do n. è. - I tysâtcheletie n. è. Moskva 1986.

Vogt 2006 - M. Vogt: Spangenhelme. Baldenheim und verwandte Typen. Mainz 2006

Werner 1953 -J. Werner: Les boucles de ceinture trouvées ans les tombes d'hommes VIII, XI, XIII, XVI et XVII. Annales de la Société archéologique de Namur 48, 1953, 320-339.

Werner 1988 - J. Werner : Neue zur Herkunft der frühmittelalterlichen Spangenhelme vom Baldenheimer Typus. Germania 66, 1988, 521-528.

Zaseckaja 1999 - I. Zaseckaja: Les steppes pontiques à l'époque hunnique. In: J. Tejral/C. Pilet/M. Kazanski (dir.) : L'Occident romain et l'Europe centrale au début de l'époque des Grandes Migrations. Brno 1999, 341-358.
Manuscript adopté le 27. 10. 2019

Abstract and keywords translated by author Súhrn preložila Hviezdoslava Zábojníková
Dr. hab. Michel Kazanski

Centre National de Recherche Scientifique

UMR 8167 Orient et Méditerranée

53, rue du Cardinal Lemoine

FR - 75005 Paris

michel.kazanski53@gmail.com 


\title{
Reprezentačné zbrane a luxusné súčasti jazdeckej výbavy v oblasti stredného a horného Dnepra v posthúnskom období
}

\author{
Michel Kazanski
}

SÚHRN

Cielom príspevku je predstavit’ „prestížne“ archeologické nálezy vojenského charakteru - zbrane a súčasti konského postroja - v oblasti stredného a horného Dnepra v posthúnskom období (druhá polovica 5. stor. - polovica 6. stor.). Nálezy zbraní a luxusných súčastí jazdeckej výbavy posthúnskeho obdobia boli nájdené v Dneperskej oblasti $\mathrm{v}$ dvoch pásmach:

- Dneper - lavý breh, medzi riekami Psel a Desna (koločinská kultúra): Kartamyševo, Klimovská oblast', Boldyžský Les, Gluchovská oblast’ (obr. 1: 1-4);

- Dneper - pravý breh, medzi riekami Stugna a Ťasmin (peňkovská kultúra), Babiči, Čerkasská oblast’ (obr. 1: 5,6$)$.

V dneperskej oblasti v posthúnskom období boli kovové predmety bežné, našli sa najmä ozdoby opaskov mužského vojenského odevu a vojenská výzbroj (obr. 8). Tieto luxusné nálezy z povodia Dnepra majú výrazne vojenský charakter: sú to prilby baldenheimského typu, meč „pontického“ typu, ozdoby sedla atd'. Je zrejmé, že tieto predmety patrili vládnucej elite bojovníkov. Nálezy luxusných zbraní, luxusné súčasti konského postroja a vojenské ozdoby (súčasti opaska) umožňujú hovorit o „vojenskej móde“ medzinárodného charakteru medzi populáciou stredného a horného Dnepra v posthúnskom období v druhej polovici 5. stor. až v polovici 6. stor. Existencia konkrétnych oblastí rozšírenia takýchto predmetov umožňuje určit’ dve hypotetické centrá moci: jedno v oblasti horného Dnepra a l'avého brehu Dnepra, niekde medzi riekami Desna a Psel, na území koločinskej kultúry a druhé na pravom brehu Dnepra, v povodí riek Ros a Tasmin, to znamená v oblasti peňkovskej kultúry (obr. 1).

Pokial' ide o písomné pramene, tieto poskytujú málo informácií o existencii vojenských elít medzi Sklavínmi a Antami. Na základe svedectiev Prokopia Cézarejského o Antoch (okolo roku 540), informácii Menandera o avarsko-antských vztahoch (okolo roku 560) a avarsko-sklavínskych vzt’ahoch (rok 578), možno dospiet’ k záveru, že slovanské vládnuce elity mali politické a vojenské funkcie. Skúmané archeologické nálezy patriace peňkovskej a koločinskej kultúre potvrdzujú existenciu týchto elít. Tieto sa pravdepodobne prvýkrát objavili medzi rokmi 536-551, ked' sa Anti a Sklavíni objavili v službách Východnej ríše a ked' sa Balkánom prehnala vlna slovanských vpádov. Predpokladá sa, že na týchto inváziách sa zúčastnilo vel'ké množstvo Slovanov, často zdaleka. Je pravdepodobné, že v dôsledku týchto udalostí padli do rúk Slovanov luxusné rímske zbrane, ako napríklad prilby baldenheimského typu alebo „pontické“ meče. 